\title{
GALERKIN SPECTRAL SYNTHESIS METHODS FOR DIFFUSION EQUATIONS WITH GENERAL BOUNDARY CONDITIONS
}

\author{
Beny Neta* \\ Department of Mathematics \\ Naval Postgraduate School \\ Code MA/Nd \\ Monterey, CA 93943 \\ U. S. A. \\ Simeon Reich \\ Department of Mathematics \\ The Technion- Israel Institute of Technology \\ 32000 Haifa \\ Israel \\ H. Dean Victory, Jr. \\ Department of Mathematics \\ Texas Tech University \\ Lubbock, TX 79409 \\ U. S. A.
}

\footnotetext{
${ }^{*}$ Corresponding author. email: bneta@nps.navy.mil, telephone: (831) 656-2235, fax: (831) 656-2355
} 


\begin{abstract}
An existence and uniqueness theory is developed for the energy dependent, steady state neutron diffusion equation with inhomogeneous oblique boundary conditions imposed. Also, a convergence theory is developed for the Galerkin Spectral Synthesis Approximations which arise when trial functions depending only on energy are utilized. The diffusion coefficient, the total and scattering cross-sectional data are all assumed to be both spatially and energy dependent. Interior interfaces defined by spatial discontinuities in the cross-section data are assumed present. Our estimates are in a Sobolev-type norm, and our results show that the spectral synthesis approximations are optimal in the sense of being of the same order as the error generated by the best approximation to the actual solution from the subspace to which the spectral synthesis approximations belong.
\end{abstract}

Subject Classification: 35J, 35Q, 65J

Keyword: Spectral synthesis, Neutron diffusion, Galerkin, Sobolev space

\title{
1 Introduction
}

As indicated in the survey article by C. H. Adams [1], synthesis methods in general have been utilized quite satisfactorily to obtain computational results for the neutron diffusion approximation to nuclear reactor problems. Such methods consist of all techniques in which an approximate solution is sought in the form

$$
\sum_{i=1}^{N} \omega_{i}(x) \psi_{i}(x, y)
$$

where $x$ is a subset of the independent variables, $y$ represents the remaining independent variables; the $\psi_{i}$ are the known trial functions, and $\omega_{i}(x)$ are the combining coefficients. The basic idea of synthesis methods is to substitute the approximation into the governing diffusion equation, take the inner product over $y$ of the resulting expressions with test functions $\xi_{i}(x, y)$, and then to determine the combining coefficients $\omega_{i}(x)$ by solving the resulting linear system. Galerkin synthesis method result when the spaces of test and trial functions are the same.

Unfortunately, however, the theoretical underpinnings of synthesis methods seem to be lacking. The research toward constructing a convergence theory for synthesis methods seems to consist of the work by Meyer and Nelson [8] and [9]. They considered the special case of Galerkin spectral synthesis approximations in which $\xi_{i}=\psi_{i}=\psi_{i}(E)$, where $E$ represents energy. In [9], these authors considered the convergence question of Galerkin spectral synthesis approximations to the continuous energy diffusion equations, in which the diffusion coefficient and the total cross-section are both spatially dependent. The convergence of the spectral synthesis approximations was strictly in an $L_{2}$-setting for functions of both space and energy, and was obtained by using operatortheoretic results of Polskij [12] concerning projection methods. In [8], on the other 
hand, spatially dependent cross-sections and diffusion coefficients were present, and the techniques employed to prove convergence were similar to those utilized in analyzing the convergence of finite element methods in approximating elliptic boundary-value problems $[4,13]$. The Hilbert space for the analysis was chosen to be an $L_{2}$ space of Sobolev space valued functions. The elements of this Hilbert space are $L_{2}$ mappings of the energy domain $(0, \infty)$ into the Sobolev space $H_{0}^{1}(\Omega)$, where $\Omega$ is the spatial domain. In working with a variational form of the diffusion equation, the authors imposed conditions on the total and scattering cross-sections which guarantee a submultiplying diffusion process in order to exploit the Lax-Milgram lemma [5] to obtain a unique weak solution to the diffusion problem. The boundary conditions were homogeneous Dirichlet conditions (in the spatial variable). The convergence of the spectral synthesis approximations to the weak solution is optimal in the sense that the errors are of the same order as the errors predicted by the best approximation to the weak solution from the subspaces where the spectral synthesis approximations occur. Neta [10] relaxed the stringent conditions imposed on the scattering cross-section data by Meyer and Nelson to guarantee convergence of the spectral synthesis approximants, but, unfortunately, the convergence results in this more genral setting were not shown to be of the "optimal type" as those of Meyer and Nelson ([8], pp. 907-908). In all of the aforementioned works, the weak solution of the diffusion problem was not shown to be classical once smoothnes assumptions on the source and cross-section data were stipulated. It is appropriate to remark here that a survey of theoretical and numerical questions in obtaining a rigorous convergence theory for synthesis methods can be found in Neta and Victory [11].

The present work considers spectral schemes with Galerkin weighting, and applies techniques used in studying projection methods [16] to examine the convergence question. As in [8], we too allow for spatial discontinuities in the diffusion coefficient and underlying cross-section data. Our work, however, extends that of Meyer and Nelson in the sense that more general boundary conditions (i.e., oblique boundary conditions) for the diffusion equation are treated. The coercivity assumption in ([8], (2.13)), involving the total and scattering cross-section data, will be weakened by observing that the scattering operator will provide a compact mapping from its domain space to its dual. We will apply the results of Witsch concerning projection methods for operators compactly perturbed ([16], pp. 343-344).

In Section 2, assumptions about the cross-section data and the diffusion coefficient are formally stated, and the existence and uniqueness questions for both classical and weak solutions to interface diffusion problems are treated. The spectral synthesis approximations are formulated in Section 3, and the existence of these approximations is settled. In Section 4, we present our convergence results, which indicate that our spectral synthesis approximations to the diffusion solution are of the same order as the best approximation to the diffusion solution from the subspaces to which the spectral synthesis solutions belong. Section 5 treats the convergence of the multigroup approximations to the diffusion equation. 


\section{The Energy-Dependent Neutron Diffusion Problem}

Let $D(x, E)$ be the diffusion coefficient, $\Sigma(x, E)$ the total cross-section, $S(x, E)$ the spontaneous source, and $k\left(x, E, E^{\prime}\right)$ the scattering kernel with $x$ an element of the spatial domain $\Omega \subset \mathcal{R}^{n}$ and the energy variable $E$ an element of the interval $(0, \infty)$. We also assume that $D$ and $\Sigma$ can be viewed as piecewise continuous mappings of $\Omega$ to $L_{\infty}((0, \infty))$ which is the Banach space of functions essentially bounded in energy, with norm $\|f\|_{\infty}=$ ess $\sup _{E \in(0, \infty)}|f(E)|$; moreover, as a function of two variables, $D(x, E)$ is bounded below by a positive constant $c_{1}$. Our scattering kernel will be viewed as a piecewise continuous mapping from $\Omega$ to $L_{2}((0, \infty) \times(0, \infty))$. The region $\Omega$ is taken to be bounded and open. Further, we assume that $\Omega$ is possibly divided into a finite number of subdomains $\Omega_{i}$, each with boundary $\partial \Omega_{i}$. Also, we assume these boundaries are sufficiently well behaved so that integration by parts may be performed. Clearly, portions of $\partial \Omega_{i}$ will make up the interfaces and the rest will make up the boundary $\partial \Omega$ of $\Omega$.

The underlying diffusion problem we consider is

$$
\begin{gathered}
\mathbf{A} \phi=\mathbf{K} \phi+S(x, E), \quad x \epsilon \Omega, E \epsilon(0, \infty) \\
\alpha \phi(x, E)+D(x, E) \frac{\partial \phi(x, E)}{\partial n}=\gamma, \quad \alpha \geq 0, x \epsilon \Omega, E \epsilon(0, \infty)
\end{gathered}
$$

where both $\phi(x, E)$ and $D(x, E) \frac{\partial \phi(x, E)}{\partial n}$ are continuous across interfaces, and where the operator $\mathbf{K}$ is defined by

$$
\mathbf{K} \phi=\int_{0}^{\infty} k\left(x, E, E^{\prime}\right) \Sigma\left(x, E^{\prime}\right) \phi\left(x, E^{\prime}\right) d E^{\prime},
$$

and the operator $\mathbf{A}$ is defined by

$$
\mathbf{A} \phi=-\nabla \cdot(D(x, E) \nabla \phi(x, E))+\Sigma(x, E) \phi(x, E) .
$$

Note that if the term containing $\frac{\partial \phi}{\partial n}$ in $(2.2)$ is not present, i.e. the boundary conditions are of Dirichlet type, the integrals over the boundary in (2.7) and (2.8b) will not be present, and the test functions must vanish on the boundary. If the boundary conditions are of Neumann type $(\alpha=0)$, then we have a boundary integral only in (2.7).

Let us introduce inner products and norms:

$$
\begin{gathered}
(u, v)=\int_{0}^{\infty} \int_{\Omega} u(x, E) v(x, E) d x d E \\
<u, v>=(u, v)+\int_{0}^{\infty} \int_{\Omega} \nabla u(x, E) \cdot \nabla v(x, E) d x d E
\end{gathered}
$$




$$
\begin{gathered}
(u, v)_{\partial \Omega}=\int_{0}^{\infty} \int_{\partial \Omega} u(x, E) v(x, E) d x d E \\
\|u\|_{0}=(u, u)^{1 / 2} \\
\mathbf{|} u \mathbf{|}=(u, u)_{\partial \Omega}^{1 / 2} \\
\|u\|_{1}=<u, u>^{1 / 2}
\end{gathered}
$$

Our analysis will primarily utilize the space $L_{2}\left((0, \infty) ; H_{0}^{1}(\Omega)\right)$, equipped with the norm (2.4f). It is easily seen that this particular space is the function space of primary importance in [8] and [10]. Also $L_{2}(\Omega \times(0, \infty))$ will denote the Hilbert space of functions square-integrable with respect to the position and energy variables equipped with the norm described in $(2.4 \mathrm{~d})$.

From our initial assumptions, we see that

$$
\left(D u_{x}, u_{x}\right) \geq c_{1}\left\|u_{x}\right\|_{0}^{2}
$$

and

$$
\left(D u_{x}, v_{x}\right)+(\Sigma u, v) \leq c_{2}\|u\|_{1}\|v\|_{1} .
$$

Concerning the scattering kernel $k$, we assume further that

$$
\begin{gathered}
\sup _{\Omega} \int_{0}^{\infty} \int_{0}^{\infty} k^{2}\left(x, E, E^{\prime}\right) \Sigma^{2}\left(x, E^{\prime}\right) d E^{\prime} d E<\infty, \\
\sup _{\Omega} \int_{0}^{\infty} \int_{0}^{\infty}\left|k\left(x+h, E+t, E^{\prime}\right) \Sigma\left(x+h, E^{\prime}\right)-k\left(x, E, E^{\prime}\right) \Sigma\left(x, E^{\prime}\right)\right|^{2} d E^{\prime} d E \rightarrow 0,
\end{gathered}
$$

as $|h|+|t| \rightarrow 0$ where $k \equiv 0$ outside $\Omega \times(0, \infty) \times(0, \infty)$;

$$
\sup _{\Omega} \int_{s}^{\infty} \int_{0}^{\infty} k^{2}\left(x, E, E^{\prime}\right) \Sigma^{2}\left(x, E^{\prime}\right) d E^{\prime} d E \rightarrow 0, \quad \text { as } s \rightarrow \infty .
$$

The mapping properties of the operator $\mathbf{K}$ defined by (2.3a) are described in the following lemma the proof of which appears in Appendix A.

Lemma 1. The operator $\mathbf{K}$ is a compact mapping of $L_{2}\left((0, \infty) ; H^{1}(\Omega)\right)$ to $L_{2}(\Omega \times$ $(0, \infty))$.

We now turn to the formulation of a weak version of the problem (2.1)-(2.2). To this end we need the following trace properties to hold, see Lions [7] and Adams ([2], pp.184-191).

Lemma 2. Assuming $\partial \Omega$ is smooth enough there is a trace operator $\gamma_{0}$ 


$$
\gamma_{0} v=\left.v\right|_{\partial \Omega \times(0, \infty)}
$$

which is linear and continuous from $L_{2}\left((0, \infty) ; H^{1}(\Omega)\right)$ into $L_{2}\left((0, \infty) ; H^{1 / 2}(\partial \Omega)\right)$. Remark: We will use $v$ instead of $\gamma_{0} v$ on $\partial \Omega$.

Weak Formulation: Find $u \epsilon L_{2}\left((0, \infty) ; H^{1}(\Omega)\right)$ such that

$$
B(u, v)=(S, v)+(\gamma, v)_{\partial \Omega}, \quad \text { for all } v \epsilon L_{2}\left((0, \infty) ; H^{1}(\Omega)\right),
$$

where

$$
\begin{gathered}
B(u, v)=a(u, v)-(\mathbf{K} u, v), \\
a(u, v)=\int_{0}^{\infty} \int_{\Omega}\{D(x, E) \nabla u(x, E) \cdot \nabla v(x, E)+\Sigma(x, E) u(x, E) v(x, E)\} d x d E \\
+\alpha \int_{0}^{\infty} \int_{\partial \Omega} u(x, E) v(x, E) d x d E,
\end{gathered}
$$

and

$$
\gamma \in L_{2}\left((0, \infty) ; H^{-1 / 2}(\partial \Omega)\right), \quad S \epsilon L_{2}(\Omega \times(0, \infty)) .
$$

Clearly the last integral in $(2.8 \mathrm{~b})$ is on the boundary $\partial \Omega$ and thus $u, v$ are the restrictions of these functions to $\partial \Omega$ (see Lemma 2).

Because $a(u, v)$ is a continuous bilinear form (the boundary term is continuous as a result of Lemma 2), we can consider A a continuous linear mapping of $L_{2}\left((0, \infty) ; H^{1}(\Omega)\right)$ to its dual, by virtue of the duality mapping

$$
a(u, v)=(\mathbf{A} u, v) .
$$

By the continuity of the bilinear form, the operator $A$ can be extended to all elements of $L_{2}\left((0, \infty) ; H^{1}(\Omega)\right)$ and have range in the dual of $L_{2}\left((0, \infty) ; H^{1}(\Omega)\right)$.

Theorem 3. Under subcritical conditions (in which 1 is not in the spectrum of $\mathbf{K}$ relative to $\mathbf{A}$ ), the weak formulation of the classical diffusion problem described by (2.7) has a unique solution $u \epsilon L_{2}\left((0, \infty) ; H^{1}(\Omega)\right)$.

Proof. Since $\Sigma(x, E)$ is positive, one obtains using (2.8b), (2.4) and Lemma 2 that

$$
\begin{gathered}
a(u, u) \geq c_{1}\left\|u_{x}\right\|_{0}^{2}+\sigma\|u\|_{0}^{2}+\alpha \mathbf{I} \mathbf{|}^{2} \geq c_{3}\|u\|_{1}^{2}, \\
a(u, v) \leq c_{2}\|u\|_{1}\|v\|_{1}+\alpha \mathbf{\|} v \mathbf{I} \leq c_{4}\|u\|_{1}\|v\|_{1} .
\end{gathered}
$$

Since $\mathbf{K}$ is compact, one obtains by the Riesz-Fredholm alternative ([4], Theorem 1-15, p. 62) that since 1 is not in the spectrum of $\mathbf{K}$ relative to $\mathbf{A}$ there exists a unique solution $u$ of $(2.7)$.

In our next theorem we show the "equivalence" between the weak and classical solution which will yield, using the Lax-Milgram theorem, the existence and uniqueness to the classical problem. 
Theorem 4. The interface problem (2.1)-(2.2) is equivalent to the weak formulation (2.7) in the sense that every solution of (2.1)-(2.2) is a solution of (2.7) and every sufficiently differentiable solution of (2.7) is a solution of (2.1)-(2.2).

Proof. Multiply $(2.1)$ by $\psi(x, E)$ and integrate over $\Omega \times(0, \infty)$ one has after applying Green's formula

$$
\begin{aligned}
& \int_{0}^{\infty} \int_{\Omega} D(x, E) \nabla \phi(x, E) \cdot \nabla \psi(x, E) d x d E+\int_{0}^{\infty} \int_{\Omega} \Sigma(x, E) \phi(x, E) \psi(x, E) d x d E \\
& -\int_{0}^{\infty} \int_{\partial \Omega} D(x, E) \nabla \phi(x, E) \cdot n \psi(x, E) d x d E=\int_{0}^{\infty} \int_{\Omega} \mathbf{K} \phi(x, E) \psi(x, E) d x d E \\
& +\int_{0}^{\infty} \int_{\Omega} S(x, E) \psi(x, E) d x d E .
\end{aligned}
$$

Using (2.2) one obtains (2.7)

$$
B(\phi, \psi)=(S, \psi)+\int_{0}^{\infty} \int_{\partial \Omega} \gamma \psi(x, E) d x d E .
$$

Conversely, let $\phi$ be a solution of (2.7) then

$$
\begin{aligned}
& \int_{0}^{\infty} \int_{\Omega} D(x, E) \nabla \phi(x, E) \cdot \nabla \psi(x, E) d x d E+\int_{0}^{\infty} \int_{\Omega} \Sigma(x, E) \phi(x, E) \psi(x, E) d x d E \\
& +\alpha \int_{0}^{\infty} \int_{\partial \Omega} \phi(x, E) \psi(x, E) d x d E-\int_{0}^{\infty} \int_{\Omega} \mathbf{K} \phi \psi(x, E) d x d E \\
& =\int_{0}^{\infty} \int_{\Omega} S(x, E) \psi(x, E) d x d E+\int_{0}^{\infty} \int_{\partial \Omega} \gamma \psi(x, E) d x d E, \forall \psi \epsilon L_{2}\left((0, \infty) ; H^{1}(\Omega)\right) .
\end{aligned}
$$

Inetgration by parts yields

$$
\begin{aligned}
& \int_{0}^{\infty} \int_{\Omega}\{-\nabla \cdot[D(x, E) \nabla \phi(x, E)]+\Sigma(x, E) \phi(x, E)-\mathbf{K} \phi-S(x, E)\} \psi(x, E) d x d E \\
& +\int_{0}^{\infty} \int_{\partial \Omega}\{-\gamma+\alpha \phi(x, E)+D(x, E) \nabla \phi(x, E) \cdot n\} \psi(x, E) d x d E=0 .
\end{aligned}
$$

If $\psi$ vanishes everywhere on $\partial \Omega$ then the boundary integral is zero and one has $(2.1)$. Returning to (2.12) one now has

$$
\int_{0}^{\infty} \int_{\partial \Omega}\{\alpha \phi(x, E)+D(x, E) \nabla \phi(x, E) \cdot n-\gamma\} \psi(x, E) d x d E=0
$$

which yields (2.2).

As a result of the equivalence theorem, we can use the Lax-Milgram theorem to prove existence and uniqueness of the solutions, assuming

$$
\left(D \frac{\partial \phi}{\partial n}, \phi\right)_{\partial \Omega} \geq 0 \quad \text { for any } \phi \epsilon L_{2}\left((0, \infty) ; H^{1 / 2}(\partial \Omega)\right),
$$

see Aubin ([4] p. 172). 


\section{Spectral Synthesis Approximation}

To specify the synthesis method, we first prescribe a set of functions

$$
\left\{\xi_{i}(E): E \epsilon(0, \infty), i=1,2, \ldots, n\right\}
$$

in $L_{2}((0, \infty))$. These functions define a subspace $W_{n} \subset L_{2}\left((0, \infty) ; H^{1}(\Omega)\right)$ given by

$$
W_{n}=\left\{\Psi \epsilon L_{2}\left((0, \infty) ; H^{1}(\Omega)\right): \Psi(x, E)=\sum_{i=1}^{n} \omega_{i}(x) \xi_{i}(E), \omega_{i} \epsilon H^{1}(\Omega), i=1,2, \ldots, n\right\} .
$$

Clearly, with respect to the topology of $L_{2}\left((0, \infty) ; H^{1}(\Omega)\right), W_{n}$ is closed in $L_{2}\left((0, \infty) ; H^{1}(\Omega)\right)$, and is thus a Hilbert space.

We seek a solution $\Psi$ in $W_{n}$ of the form

$$
\Psi(x, E)=\sum_{i=1}^{n} \omega_{i}(x) \xi_{i}(E) .
$$

The weights $\omega_{i}(x)$ are determined by substituting (3.2) into the following weak analog of (2.7). Find a $\Psi \in W_{n}$ such that

$$
B(\Psi, \zeta)=(S, \zeta)+(\gamma, \zeta)_{\partial \Omega}, \text { for all } \zeta \epsilon W_{n}
$$

Theorem 5. The approximate problem (3.3) has a unique solution $\Psi \epsilon W_{n}$.

Proof. Since $W_{n}$ is a subspace of $L_{2}\left((0, \infty) ; H^{1}(\Omega)\right)$, we can follow the steps of the proof of Theorem 3 to get the existence and uniqueness of solutions of the approximate problem.

To get another proof of uniqueness, we reformulate (3.3) as

$$
((\mathbf{A}-\mathbf{K}) \Psi, \zeta)=(S, \zeta)+(\gamma, \zeta)_{\partial \Omega}, \text { for all } \zeta \epsilon W_{n} .
$$

where the operator $\mathbf{A}$ is given by (2.9). It is easy to see, from Lemma 1, that the operator $\mathbf{A}^{-1} \mathbf{K}$ will be indeed a compact operator on $L_{2}\left((0, \infty) ; H^{1}(\Omega)\right)$.

We now show that the spectral synthesis approximations for $\mathbf{B}=\mathbf{A}-\mathbf{K}$ effects a projection method in the sense of Witsch [16]. Indeed, from (2.10), we have that $\mathbf{A}$ is $L_{2}\left((0, \infty) ; H^{1}(\Omega)\right)$-elliptic, i.e.

$$
(\mathbf{A} u, u) \geq c_{3}\|u\|_{1}^{2} .
$$

Hence, not only $\mathbf{A}$ is a bounded mapping from $L_{2}\left((0, \infty) ; H^{1}(\Omega)\right)$ to its dual, but, to each $u \epsilon W_{n}$, there corresponds a $v \epsilon W_{n}$, namely $v=u$, such that

$$
(\mathbf{A} u, u) \geq c_{3}\|u\|_{1}^{2} .
$$

Therefore the projection method for $\mathbf{A}$ satisfies the criterion

$$
u \epsilon W_{n},(\mathbf{A} u, v)=0 \text { for all } v \epsilon W_{n} \text { implies } u=0 .
$$


Since $\mathbf{K}$ is a compact operator and 1 is not in the spectrum of $\mathbf{K}$ relative to $\mathbf{A}$ we have a similar result for $\mathbf{B}=\mathbf{A}-\mathbf{K}$, i.e.

$$
u \epsilon W_{n},(\mathbf{B} u, v)=0 \text { for all } v \epsilon W_{n} \text { implies } u=0 .
$$

We can use this as an alternative proof of uniqueness. Now suppose the problem doesn't have a unique solution, i.e. we have two solutions $w_{1}$ and $w_{2}$. Let $u=w_{1}-w_{2}$, then (3.5) applied to the operator $\mathbf{B}$ implies that $u=0$ or $w_{1}=w_{2}$, and thus the solution is unique.

\section{Convergence Results}

In this section we show that under the assumptions in Section 2, the spectral synthesis approximations to the diffusion problem are of the same order as the best approximation to the diffusion solution from the subspaces $W_{n}$.

Theorem 6. Let $u$ be the solution of (2.7) and $\phi_{n}$ be the solution of (3.3). Let $\hat{\phi}_{n}$ be the best approximation of the solution $u$ from the subspaces $W_{n}$. Then

$$
\left\|u-\phi_{n}\right\|_{1} \leq M\left\|u-\hat{\phi}_{n}\right\|_{1}
$$

Proof. Using (3.5) we can conclude (see Witsch [16]) that there will be a projection operator associated with $W_{n}$, which we shall denote $\boldsymbol{\Pi}_{n}$. So our problem becomes equivalent to

$$
u_{n} \epsilon W_{n}, \boldsymbol{\Pi}_{n}\left(\mathbf{B} u_{n}-S\right)=0,
$$

and

$$
u_{n}=\mathbf{A}^{-1}\left(\mathbf{P}_{n} S\right)=\mathbf{Q}_{n}\left(\mathbf{A}^{-1} S\right)=\mathbf{Q}_{n} u
$$

with

$$
\mathbf{P}_{n}=\left(\left.\boldsymbol{\Pi}_{n}\right|_{\mathbf{A}\left(\mathbf{W}_{\mathbf{n}}\right)}\right)^{-1} \mathbf{\Pi}_{n}, \mathbf{Q}_{n}=\left(\left.\boldsymbol{\Pi}_{n} \mathbf{A}\right|_{W_{n}}\right)^{-1} \boldsymbol{\Pi}_{n} \mathbf{A}
$$

See the diagram in Section 2.1 of Witsch [16]. The projections $\mathbf{P}_{n}$ are especially adapted for investigations concerning the convergence of the residual $\mathbf{A} u_{n}-S$ (consistency) and the $\mathbf{Q}_{n}$ are for the convergence of $u_{n}$ to $u$. We apply Theorem 2.2 of Witsch [16] to conclude that we have consistency and convergence.

The question now is, can we prove the same for the operator B. From Theorem 2.6 of Witsch [16], we know that the $\mathbf{Q}_{n}$ for $(\mathbf{A}-\mathbf{K})$ are defined for sufficiently large $n$, and are uniformly bounded. Hence we get that $\phi_{n}$, given by the spectral synthesis method, will converge to $u$ in the topology of $L_{2}\left((0, \infty) ; H^{1}(\Omega)\right)$, and moreover, $\phi_{n}=\mathbf{Q}_{n} u$ Now to the error estimate

$$
\begin{aligned}
\left\|u-\phi_{n}\right\|_{1} & \leq\left\|u-\hat{\phi}_{n}\right\|_{1}+\left\|\hat{\phi}_{n}-\phi_{n}\right\|_{1} \\
& \leq\left\|u-\hat{\phi}_{n}\right\|_{1}+\left\|\mathbf{Q}_{n}\left(\hat{\phi}_{n}-u\right)\right\|_{1} \\
& \leq\left(1+\left\|\mathbf{Q}_{n}\right\|_{1}\right)\left\|u-\hat{\phi}_{n}\right\|_{1}
\end{aligned}
$$


The norm of $\mathbf{Q}_{n}$ can be estimated by

$$
\begin{aligned}
\left\|\mathbf{Q}_{n}\right\| & \leq\left\|\mathbf{Q}_{n} \mathbf{B}^{-1}\right\|\|\mathbf{B}\| \leq \sup _{x \in W_{n}} \inf _{y^{*} \in W_{n}} \frac{\left\|y^{*}\right\|\|x\|}{\left|\left(y^{*}, \mathbf{B} x\right)\right|}\|\mathbf{B}\| \\
& \leq \sup _{x \in W_{n}} \frac{\|x\|^{2}}{c_{3}\|x\|^{2}}\|\mathbf{B}\| \leq \frac{\|\mathbf{B}\|}{c_{3}} .
\end{aligned}
$$

Using this estimate in (4.2), we have

$$
\left\|u-\phi_{n}\right\|_{1} \leq\left(1+\frac{\|\mathbf{B}\|}{c_{3}}\right)\left\|u-\hat{\phi}_{n}\right\|_{1}
$$

\section{The Multigroup Method}

The multigroup method is perhaps the most widely used technique for approximately determining the energy distribution of particles in a system modeled by linear transport [14]. It is usually assumed that the solutions of the multigroup equations approximate the corresponding solution of the exact transport equation in which the energy variable is not discretized. In spite of the wide usage, there does seem to be a dearth of work concerned with studies of how the multigroup solutions approximate the exact solution and with an evaluation of the sources of the errors incurred in using the method $[14,15]$. One of the authors [14] has discussed the convergence of the multigroup approximation fo multidimensional media. Here we are only interested in the spectral synthesis approximation.

In the multigroup method, the flux is assumed to be separable into a product of a known function dependent on energy and position and a function dependent on position and direction over each of the specified energy intervals. This approach leads to a set of coupled multigroup transport equations that describe the behavior of the multigroup fluxes as functions of position and direction, see Allen et al [3]. In this case the operator $\mathbf{K}$ is

$$
\mathbf{K} \phi=\int_{E_{0}}^{E_{\max }} k\left(x, E, E^{\prime}\right) \Sigma\left(x, E^{\prime}\right) \phi\left(x, E^{\prime}\right) d E^{\prime},
$$

Let $\Pi_{G}$ be a partition of $\left[E_{0}, E_{\max }\right] \subset(0, \infty)$ specified by

$$
0=E_{0}<E_{1}<\ldots<E_{G}=E_{\max }
$$

with $E_{0}$ and $E_{\max }$, respectively, denoting the minimum and maximum energies attainable by a particle, and $G$ is the number of energy groups. Let $\left\{\psi_{g}(E), g=1,2, \ldots, G\right\}$ be a set of functions in $L_{2}((0, \infty))$ satisfying

$$
\psi_{g}(E)=\left\{\begin{array}{ll}
1 & E \epsilon\left[E_{g-1}, E_{g}\right) \\
0 & \text { otherwise }
\end{array}, g=1,2, \ldots, G .\right.
$$

Let $W_{G}$ be the space of functions defined by

$$
W_{G}=\left\{v \epsilon L_{2}\left((0, \infty) ; H^{1}(\Omega)\right) ; v(x, E)=w_{g}(x, E) \psi_{g}(E), 1 \leq g \leq G\right\} .
$$


We now define our multigroup solution $\eta(x, E) \epsilon W_{G}$ and require that $\eta(x, E)$ satisfies the following system of $G$ equations for all $v \epsilon W_{G}$

$$
\begin{aligned}
& \int_{E_{0}}^{E_{G}} \int_{\Omega}\{-\nabla \cdot[D(x, E) \nabla \eta(x, E)]+\Sigma(x, E) \eta(x, E)-\mathbf{K} \eta\} w_{g}(x, E) \psi_{g}(E) d x d E \\
& =\int_{E_{0}}^{E_{G}} \int_{\Omega} S(x, E) w_{g}(x, E) \psi_{g}(E) d x d E, 1 \leq g \leq G .
\end{aligned}
$$

As a result of (5.1), we can rewrite the above system as follows:

$$
\begin{aligned}
& \int_{E_{g-1}}^{E_{g}} \int_{\Omega}\{-\nabla \cdot[D(x, E) \nabla \eta(x, E)]+\Sigma(x, E) \eta(x, E)-\mathbf{K} \eta\} w_{g}(x, E) d x d E \\
& =\int_{E_{g-1}}^{E_{g}} \int_{\Omega} S(x, E) w_{g}(x, E) d x d E, 1 \leq g \leq G .
\end{aligned}
$$

It is clear that (5.4) is equivalent to

$$
B\left(\eta, \psi_{g}\right)=\left(S, \psi_{g}\right)+\left(\gamma, \psi_{g}\right)_{\partial \Omega}, g=1,2, \ldots, G
$$

where now the integration is on a finite energy interval. For our spectral synthesis method we seek a solution $\Psi(x, E) \epsilon W_{n, G} \subset W_{G}$ of the form

$$
\sum_{i=1}^{n} \sum_{g=1}^{G} w_{i g}(x) \psi_{i g}(E)
$$

where we let

$$
W_{n, G}=\left\{\psi \epsilon L_{2}\left((0, \infty) ; H^{1}(\Omega)\right) ; \psi(x, E)=\sum_{i=1}^{n} \sum_{g=1}^{G} w_{i g}(x) \psi_{i g}(E)\right\} .
$$

The $w_{i g}(x)$ are determined by substituting (5.6) into (2.1) and then projecting onto the space spanned by the functions $\psi_{i g}(E)$. Thus $\Psi$ must satisfy

$$
B\left(\Psi, \psi_{g}\right)=\left(S, \psi_{g}\right)+\left(\gamma, \psi_{g}\right)_{\partial \Omega}, \text { for all } \psi_{g} \in W_{n, G} \text { and } g=1,2, \ldots, G \text {. }
$$

Note the similarity between (5.5), (5.8) and (2.7), (3.3) respectively. The only difference is that the energy integration is on a finite interval. Therefore one obtains results similar to theorems 5 and 6 .

Theorem 7. The approximate system (5.8) has a unique solution in $W_{n, G}$.

Theorem 8. Let $\eta$ be the solution of (5.5) and $\phi$ be the solution of (5.8). Let $\hat{\phi}$ be the best approximation of the solution $\eta$ from the subspaces $W_{n, G}$. Then

$$
\|\eta-\phi\|_{1} \leq M\|\eta-\hat{\phi}\|_{1}
$$




\section{Appendix A}

\section{Proof of Lemma 1}

Condition(2.5d) assures us that the mapping $\mathbf{K}$ will be a bounded mapping of $L_{2}\left((0, \infty) ; H^{1}(\Omega)\right)$ to $L_{2}(\Omega \times(0, \infty))$. From the Frechet-Kolmogorv theorem ([6], p. $97)$, we see that we must show, for all $u \in \mathcal{B} \equiv\left\{u \in L_{2}\left((0, \infty) ; H^{1}(\Omega)\right):\|u\|_{1} \leq 1\right\}$

$$
\begin{gathered}
(a) \sup _{\mathcal{B}} \int_{\Omega} \int_{0}^{\infty}|\mathbf{K} u(y, E)|^{2} d E d y<\infty ; \\
(b) \int_{\Omega} \int_{0}^{\infty}|\mathbf{K} \tilde{u}(y+x, E+t)-\mathbf{K} \tilde{u}(y, E)|^{2} d E d y \rightarrow 0,
\end{gathered}
$$

uniformly for $u \in \mathcal{B}$ as $|x|+|t| \rightarrow 0$, where $\tilde{u}$ is the extension of $u$ to all of $\mathcal{R}^{n+1}$ which has the value zero outside the set $\Omega \times(0, \infty)$,

$$
\text { (c) } \lim _{\alpha \rightarrow \infty} \int_{\Omega} \int_{\alpha}^{\infty}|\mathbf{K} u(y, E)|^{2} d E d y=0,
$$

uniformly for $u \in \mathcal{B}$.

To prove (a), we use the Schwarz inequality, and obtain

$$
\begin{aligned}
& \int_{\Omega} \int_{0}^{\infty}\left|\int_{0}^{\infty} k\left(y, E, E^{\prime}\right) \Sigma\left(y, E^{\prime}\right) u\left(y, E^{\prime}\right) d E^{\prime}\right| d E d y \leq \\
& \int_{\Omega} \int_{0}^{\infty}\left[\left\{\int_{0}^{\infty} k^{2}\left(y, E, E^{\prime}\right) \Sigma^{2}\left(y, E^{\prime}\right) d E^{\prime}\right\}\left\{\int_{0}^{\infty} u^{2}\left(y, E^{\prime}\right) d E^{\prime}\right\}\right] d E d y \leq \\
& \sup _{\Omega}\left[\int_{0}^{\infty} \int_{0}^{\infty} k^{2}\left(y, E, E^{\prime}\right) \Sigma^{2}\left(y, E^{\prime}\right) d E^{\prime} d E\right]\|u\|_{1}^{2} .
\end{aligned}
$$

In order to prove (b), we must extend $u \epsilon L_{2}\left((0, \infty) ; H^{1}(\Omega)\right)$ to a function belonging to $L_{2}\left((0, \infty) ; H^{1}\left(\mathcal{R}^{n}\right)\right)$. We say that $\Gamma$ is an extension operator with respect to $\Omega$ provided there exists a constant $\nu$ such that for every $u \epsilon L_{2}\left((0, \infty) ; H^{1}(\Omega)\right)$

$$
\text { (i) } \quad \Gamma u(x, E)=u(x, E), \quad x \epsilon \Omega
$$

(ii) $\quad\|\Gamma u\|_{1, \mathcal{R}^{n}} \leq \nu\|u\|_{1}$

where $\|\cdot\|_{1, \mathcal{R}^{n}}$ is the norm in $L_{2}\left((0, \infty) ; H^{1}\left(\mathcal{R}^{n}\right)\right)$ given by $(2.4 \mathrm{~d})$. Also, we note that the results in ([2], pp.84-94) concerning the extension operators for Sobolev spaces of real- or complex-valued functions, with domain $\Omega \subset \mathcal{R}^{n}$ possessing the uniform cone property ([2], pp.65-70), can be modified to produce the existence of extension operators for Sobolev spaces of Banach space- or Hilbert space-valued functions defined on similar domains. In particular, the Calderon Extension theorem ([2], pp.91-94) 
can be generalized in a straightforward manner to prove the existence of extension operators with respect to general $\Omega \subset \mathcal{R}^{n}$ for functions in $L_{2}\left((0, \infty) ; H^{p, m}(\Omega)\right)$, where

$$
H^{p, m}(\Omega)=\left\{f \in L_{p}(\Omega): f^{(i)} \epsilon L_{p}(\Omega), i=0,1, \ldots, m\right\} .
$$

More precisely, we have

The Calderon Extension theorem: Let $\Omega$ be a domain in $\mathcal{R}^{n}$ having the uniform cone property ([2], p. 66) modified as follows:

1. The open cover $\left\{U_{j}\right\}$ of the boundary of $\Omega$ is required to be finite, and

2. The sets $U_{j}$ are not required to be bounded.

Then for any $m$, and any $p, 1<p<\infty$, there exists an extension operator $\Gamma=$ $\Gamma(m, p)$ with respect to $\Omega$ for functions in $L_{2}\left((0, \infty) ; H^{p, m}(\Omega)\right)$.

Henceforth we shall let $\bar{u}(x, E)=\Gamma u(x, E), \quad(x, E) \epsilon \mathcal{R}^{n} \times(0, \infty)$. To show (b), we have with $k_{1}\left(x, E, E^{\prime}\right)=k\left(x, E, E^{\prime}\right) \Sigma\left(x, E^{\prime}\right)$,

$$
\begin{aligned}
& \int_{\Omega} \int_{0}^{\infty}|\mathbf{K} \tilde{u}(x+y, E+t)-\mathbf{K} \tilde{u}(y, E)|^{2} d E d y \leq \\
& 2 \int_{\Omega} \int_{0}^{\infty}\left|\int_{0}^{\infty} \tilde{k_{1}}\left(x+y, E+t, E^{\prime}\right)\left[\bar{u}\left(x+y, E^{\prime}\right)-\bar{u}\left(y, E^{\prime}\right)\right] d E^{\prime}\right|^{2} d E d y+ \\
& 2 \int_{\Omega} \int_{0}^{\infty}\left|\int_{0}^{\infty}\left[\tilde{k_{1}}\left(x+y, E+t, E^{\prime}\right)-\tilde{k}\left(y, E, E^{\prime}\right)\right] u\left(y, E^{\prime}\right) d E^{\prime}\right|^{2} d E d y \leq \\
& 2 \int_{\Omega}\left\{\int_{0}^{\infty} \int_{0}^{\infty}\left|\tilde{k_{1}}\left(x+y, E+t, E^{\prime}\right)\right|^{2} d E^{\prime} d E\right\} . \\
& \left\{\int_{0}^{\infty}\left|\bar{u}\left(x+y, E^{\prime}\right)-u\left(y, E^{\prime}\right)\right|^{2} d E^{\prime}\right\} d y+ \\
& 2 \int_{\Omega}^{\infty}\left\{\int_{0}^{\infty}\left|\tilde{k_{1}}\left(x+y, E+t, E^{\prime}\right)-k_{1}\left(y, E, E^{\prime}\right)\right|^{2} d E^{\prime}\right\} \\
& \left\{\int_{0}^{\infty}\left|u\left(y, E^{\prime}\right)\right|^{2} d E^{\prime}\right\} d E d y .
\end{aligned}
$$

To ascertain that

$$
\int_{\Omega} \int_{0}^{\infty}\left|\bar{u}\left(x+y, E^{\prime}\right)-u\left(y, E^{\prime}\right)\right|^{2} d E^{\prime} d y \rightarrow 0
$$

uniformly in $u \epsilon \mathcal{B}$, as $|x| \rightarrow 0$, we estimate this integral. If $u \epsilon L_{2}\left((0, \infty) ; C_{0}^{\infty}\left(\mathcal{R}^{n}\right)\right)$, we can deduce ([2], p. 186), by virtue of the absolute continuity of $u$ as a mapping of $\mathcal{R}^{n}$ 
to $L_{2}((0, \infty))$ :

$$
\begin{aligned}
& \int_{\Omega} \int_{0}^{\infty}\left|u\left(x+y, E^{\prime}\right)-u\left(y, E^{\prime}\right)\right|^{2} d E^{\prime} d y= \\
& \int_{\Omega} \int_{0}^{\infty}\left|\int_{0}^{1} \frac{\partial}{\partial t} u\left(y+t x, E^{\prime}\right) d t\right|^{2} d E^{\prime} d y= \\
& \int_{\Omega} \int_{0}^{\infty}\left|\int_{0}^{1} \frac{\partial}{\partial y} u\left(y+t x, E^{\prime}\right) x d t\right|^{2} d E^{\prime} d y \leq \\
& \int_{\Omega} \int_{0}^{\infty} \int_{0}^{1}|x|\left|\frac{\partial}{\partial y} u\left(y+t x, E^{\prime}\right)\right|^{2} d t d E^{\prime} d y \leq \\
& |x| \int_{0}^{1} d t \int_{\mathcal{R}^{n}} d y \int_{0}^{\infty}\left|\frac{\partial}{\partial y} u\left(y, E^{\prime}\right)\right|^{2} d E^{\prime} \leq|x|\|u\|_{1, \mathcal{R}^{n}}
\end{aligned}
$$

Because $L_{2}\left((0, \infty) ; C_{0}^{\infty}\left(\mathcal{R}^{n}\right)\right)$ is dense in $L_{2}\left((0, \infty) ; H^{1}\left(\mathcal{R}^{n}\right)\right)$, we have the above inequality holding for any $u \epsilon L_{2}\left((0, \infty) ; H^{1}\left(\mathcal{R}^{n}\right)\right)$ so, for $|x| \rightarrow 0$ and $u \epsilon \mathcal{B}$, we deduce by virtue of the Calderon Extension theorem,

$$
\int_{\Omega} \int_{0}^{\infty}\left|\bar{u}\left(x+y, E^{\prime}\right)-u\left(y, E^{\prime}\right)\right|^{2} d E^{\prime} d y \rightarrow 0
$$

uniformly as $|x| \rightarrow 0$.

Finally (c) follows from the inequalities,

$$
\begin{aligned}
& \int_{\Omega} \int_{\alpha}^{\infty}\left|\int_{0}^{\infty} k\left(x, E, E^{\prime}\right) \Sigma\left(x, E^{\prime}\right) u\left(x, E^{\prime}\right) d E^{\prime}\right|^{2} d E d x \leq \\
& \int_{\alpha}^{\infty} \int_{\Omega}\left\{\int_{0}^{\infty} k^{2}\left(x, E, E^{\prime}\right) \Sigma^{2}\left(x, E^{\prime}\right) d E^{\prime}\right\}\left\{\int_{0}^{\infty} u^{2}\left(x, E^{\prime}\right) d E^{\prime}\right\} d x d E \leq \\
& \left(\sup _{\Omega} \int_{\alpha}^{\infty} \int_{0}^{\infty} k^{2}\left(x, E, E^{\prime}\right) \Sigma^{2}\left(x, E^{\prime}\right) d E^{\prime}\right)\|u\|_{1} .
\end{aligned}
$$

We can deduce that the mapping $\mathbf{K}$ is compact. 


\section{References}

[1] C.H. Adams, 'Current trends in methods for neutron diffusion calculations', Nucl. Sci. Eng., 64, 552-562 (1977).

[2] R. A. Adams, Sobolev Spaces, Academic Press, New York, 1975.

[3] E. J. Allen, H. D. Victory, Jr, and K. Ganguly, 'On the convergence of finitedifferenced multigroup, discrete-ordinates methods for anisotropically scattering slab media', SIAM J. Numer. Anal., 26, 88-106, (1989).

[4] J. P. Aubin, Approximation of Elliptic Boundary-Value Problems, Wiley Interscience, New York 1972.

[5] A. Friedman, Partial Differential Equations, Holt, Reinhart and Wilson Inc., New York, 1969.

[6] M. A. Krasnosel'skii, Ya. B. Rutickii, Convex Functions and Orlicz Spaces, Noordhoff, Netherlands, 1961. (Translated from Russian by L. F. Boron).

[7] J. L. Lions, Private Communication, 1981.

[8] H. D. Meyer and P. Nelson, 'Error analysis and convergence for the spectral synthesis method with interfaces', J. Appl. Math. Phys., 30, 901-912 (1979).

[9] P. Nelson and H. D. Meyer, 'Convergence results and asymptotic error estimates for Galerkin-type spectral synthesis', Nucl. Sci. Eng., 64, 638-643 (1977).

[10] B. Neta, 'Note on error analysis for the spectral synthesis method with interfaces', ZAMP, 32, 603-608 (1981).

[11] B. Neta and H. D. Victory, 'On the development of a convergence theory of synthesis methods for solving diffusion equations', Progress Nucl. Energy, 8, 283-293 (1981).

[12] N. I. Polskij, 'Projective methods in applied mathematics', Sov. Math., 3, 488-492 (1962).

[13] G. Strang and G. J. Fix, An Analysis of the Finite Element Method, Prentice Hall, Englewood Cliffs, 1973.

[14] H. D. Victory, Jr, 'On the convergence of the multigroup approximation for multidimensional media', Annali di Matematica Pura ed Applicata, 140, (1985), 179207.

[15] H. D. Victory, Jr, E. J. Allen, 'On the convergence of the multigroup, discreteordinates solutions for subcritical transport media', Annali di Matematica Pura ed Applicata, 153, (1988), 229-274.

[16] K. Witsch, 'Convergence of projection methods for linear operators', Numer. Math. , 27, 339-354 (1977). 